\title{
Quantitative Theory for the Diffusive Dynamics of Liquid Condensates
}

\author{
Lars Hubatsch ${ }^{1,2,3}$, Louise M. Jawerth ${ }^{1,2}$, Celina Love ${ }^{2}$, Jonathan Bauermann ${ }^{1}$, T.-Y. Dora Tang ${ }^{2}$, \\ Stefano Bo ${ }^{1}$, Anthony A. Hyman ${ }^{2,3}$, Christoph A. Weber ${ }^{1,2,3, *}$ \\ ${ }^{1}$ Max Planck Institute for the Physics of Complex Systems, Nöthnitzer Strasse 38, 01187 Dresden, Germany \\ ${ }^{2}$ Max Planck Institute of Molecular Cell Biology and Genetics, Pfotenhauerstrasse 108, 01307 Dresden, \\ Germany \\ ${ }^{3}$ Center for Systems Biology Dresden, Pfotenhauerstrasse 108, 01307 Dresden, Germany \\ *For correspondence: weber@pks.mpg.de (CAW)
}

\begin{abstract}
To unravel the biological functions of membraneless liquid condensates it is crucial to develop a quantitative understanding of the physics underlying their dynamics. Key processes within such condensates are diffusion and material exchange with their environment. Experimentally, diffusive dynamics are typically probed via fluorescent labels. However, to date we lack a physics-based quantitative framework for the dynamics of labeled condensate components. Here, we derive the corresponding theory, building on the physics of phase separation, and quantitatively validate this framework via experiments. We show that using our theory we can precisely determine diffusion coefficients inside liquid condensates via a spatio-temporal analysis of fluorescence recovery after photobleaching (FRAP) experiments. We showcase the accuracy and precision of our approach by considering space- and time-resolved data of protein condensates and two different polyelectrolyte-coacervate systems. Strikingly, our theory can also be used to determine the diffusion coefficient in the dilute phase and the partition coefficient, without relying on fluorescence measurements in the dilute phase. This bypasses recently described quenching artefacts in the dense phase, which can underestimate partition coefficients by orders of magnitude. Our experimentally verified theory opens new avenues for theoretically describing molecule dynamics in condensates, measuring concentrations based on the dynamics of fluorescence intensities and quantifying rates of biochemical reactions in liquid condensates.
\end{abstract}

\section{Introduction}

Liquid phase separation has emerged as an organizing principle in biology and is thought to underlie the formation of various membrane-less cellular organelles (Banani et al. (2017)). Hallmark properties of such organelles are their rapid formation and dissolution, their fusion, and their wetting to membranes (Hyman et al. (2014)). Moreover, phaseseparated organelles exchange material with their environment leading to dynamic sequestration of molecules, which affects biochemical processes by spatial redistribution of reactants (Moon et al. (2019); Lyon et al. (2020); Saha et al. (2016); Guillén-Boixet et al. (2020); Sanders et al. (2020); Yang et al. (2020)). Probing the dynamics of condensate components is thus imperative for a quantitative understanding of how they affect the cellular biochemistry (Mir et al. (2019)).

To probe the dynamics of condensates, biomolecules are typically labelled with fluorescent tags. In general, in systems with tagged molecules, various methods exist to characterize molecular properties such as binding rates and diffusion coefficients, including fluorescence correlation spectroscopy (FCS) (Ries and Schwille (2012); Rigler and Elson (2012), single-particle tracking (SPT) (Tinevez et al. (2017); Saxton and Jacobson (1997)), and fluorescence recovery after photobleaching (FRAP) (Diaspro (2010); Stasevich et al. (2010)). However, interpretation of the experimental data acquired from such methods requires a rigorous derivation accounting for the underlying physicochemcial processes. This derivation has been achieved for some biological systems and processes, but is lacking for condensates formed by liquid 
phase separation. Processes that are well-understood include membrane-cytoplasmic exchange and transport (Sprague et al. (2004); Robin et al. (2014); Goehring et al. (2010)) as well as chemical reactions (Elson (2001)) or filament turnover (McCall et al. (2019)). For liquid condensates, various phenomenological fit functions have been proposed in the literature (e.g. Patel et al. (2015); Banerjee et al. (2017); Hubstenberger et al. (2013), for a broader summary see Taylor et al. (2019)). However, it was recently shown that these fits lead to wildly differing estimates of the diffusion constant inside, $D_{\text {in }}$ (Taylor et al. (2019)). Taylor et al. showed that these discrepancies were attributed to unrealistic assumptions, e.g. infinitely large droplets or infinitely fast diffusion outside the bleach area. However, a conclusive answer of what governs the dynamics of molecules across a condensate interface could not be reached.

Here, we first introduce a quantitative FRAP method to extract the diffusion coefficient inside $D_{\text {in }}$, purely based on fluorescence measurements inside droplets, without resorting to unrealistic assumptions or requiring knowledge about the partition coefficient, $P$ or diffusion outside, $D_{\text {out }}$. Using non-equilibrium thermodynamics, we then derive the theory that connects dynamics inside and outside of the droplet via transport across a finite interface. We show that this theory fits our experimentally observed dynamics with striking agreement. Surprisingly, we theoretically show that it can be used to extract all relevant parameters of the system, $P, D_{\text {in }}$ and $D_{\text {out }}$, purely based on knowledge of the dynamics inside the droplet. We show that our measurements are agnostic to breaking radial symmetry, for example by introducing a coverslip or neighbouring droplets. Our approach does not suffer from typical limitations of fluorescence-based concentration measurements, such as low fluorescence in the dilute phase and fluorophore quenching in the rich phase. We anticipate that this new understanding will open the door to characterizing dynamical properties such as chemical rates and rheological parameters in multi-component, phase-separated systems.

\section{Results}

\section{Determining the diffusion constant inside liquid condensates}

First, we discuss a quantitative method to extract diffusion coefficients of biomolecules in a condensate. After bleaching a liquid condensate, bleached molecules diffuse out and unbleached molecules diffuse into the condensate, until the unbleached components reach the spatially homogeneous level prior to bleaching (Fig. 1a). Inside a spherical condensate of radius $R$, the concentration of unbleached components, $c_{u}(r, t)$, follows a diffusion equation (for derivation, see subsequent section),

$$
\begin{aligned}
\partial_{t} c_{u}(r, t) & =-\nabla \cdot j_{u}, \\
j_{u} & =-D_{\mathrm{in}} \nabla c_{u}, \\
c_{u}\left(r=R_{-}, t\right), & r=R,
\end{aligned}
$$

where $c_{u}\left(r=R_{-}, t\right)$ is the time-dependent concentration directly inside the interface at $r=R_{-}$. Here, $r$ denotes the radial distance to the center of the condensate. The flux $\boldsymbol{j}_{u}$ is given by Fick's law (Eq. (1b)). It vanishes at the condensate center. Moreover, we have $\nabla=e_{r} \partial_{r}$, with $\boldsymbol{e}_{r}$ denoting the radial unit vector. During FRAP, the concentration at the interface, $c_{u}\left(r=R_{-}, t\right)$, changes with time (Fig. 1b) and is determined by the physical properties of the condensate environment. This environment is characterized by the diffusion constant and the concentration of unbleached components outside, the distribution of neighbouring condensates as well as system boundaries like the coverslip.

To initially bypass this complex dependence on the condensate environment, we propose to extract the fluorescence concentration of unbleached molecules directly inside of the condensate-bulk boundary $c_{u}\left(r=R_{-}, t\right)$ from experimental data after photobleaching (Fig. 1a,b). Using this experimentally determined dynamic boundary condition, we can accurately determine the diffusion constant inside a condensate, $D_{\text {in }}$ (Fig. 1d,f). Following this idea, we fit the solutions of Eq. (1) to spatio-temporal experimental data, with $D_{\text {in }}$ as the only fit parameter. We find excellent agreement between experimentally measured and fitted concentration profiles (Fig. 1c,e and supp. movies 1,2,3). Specifically, we consider condensates composed of PGL-3, a main protein component of P granules in the C. elegans embryo (Brangwynne et al. (2009); Griffin (2015)), as well as two synthetic polyelectrolye-complex coacervate systems, Polylysine/ATP (PLYS/ATP) and Carboxymethyldextran/Polylysine (CMD/PLYS).

We first compared $D_{\text {in }}$ of PGL-3 for different salt concentrations between $50 \mathrm{mM}$ and $180 \mathrm{mM}$ (see Methods). We find that $D_{\text {in }}$ varies by roughly one order of magnitude, between $0.009 \mu \mathrm{m}^{2} \mathrm{~s}^{-1}$ and $0.070 \mu \mathrm{m}^{2} \mathrm{~s}^{-1}$. Our trend is in good agreement with reported measurements of the viscosity $\eta$, determined by active micro-rheology (Jawerth et al. (2018)) 
bioRxiv preprint doi: https://doi.org/10.1101/2021.03.08.434288; this version posted March 8, 2021. The copyright holder for this preprint (which was not certified by peer review) is the author/funder, who has granted bioRxiv a license to display the preprint in perpetuity. It is made available under aCC-BY-NC 4.0 International license.

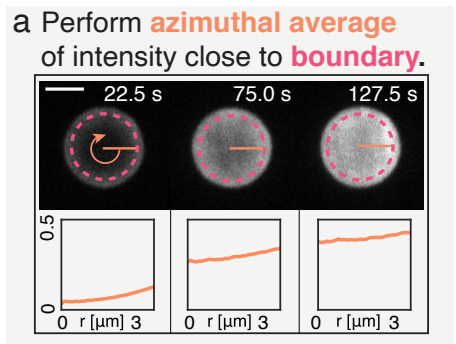

b

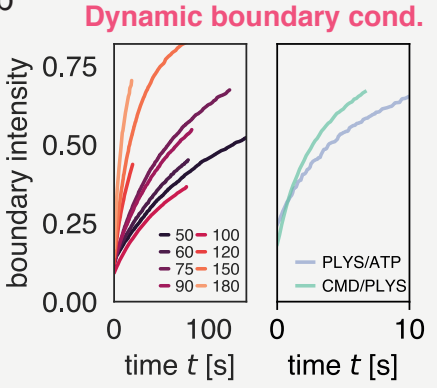

C

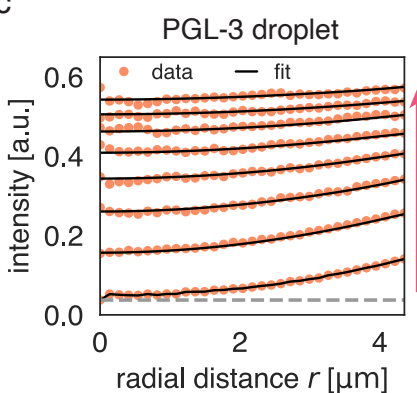

d

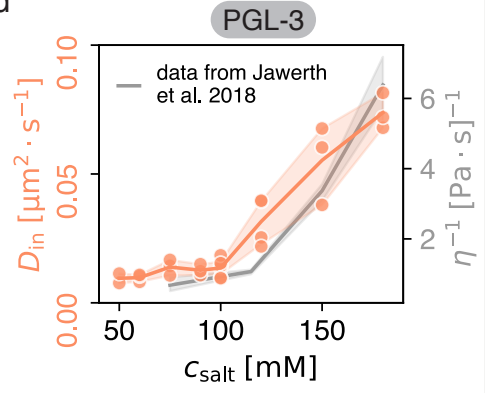

e

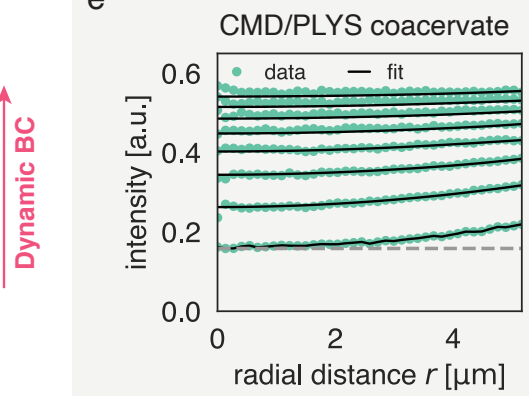

f

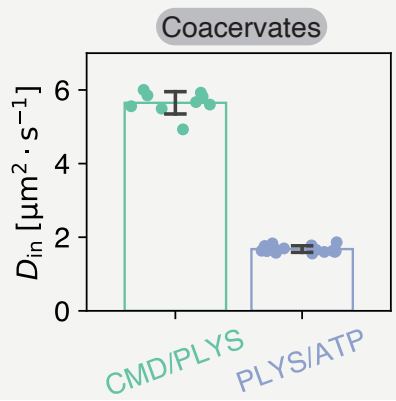

Figure 1. Quantitatively measuring $D_{\text {in }}$ by extracting concentration at condensate interface. (a) Time course of FRAP recovery after full bleach for PLYS/ATP coacervate droplet. Droplet interface (magenta) and azimuthal average (orange) are highlighted. Note, data close to the droplet boundary cannot be fit due to optical artefacts giving rise to an artificially broad interface. Thus, fluorescence intensity is extracted approximately $1.4 \mu \mathrm{m}$ away from the interface (see methods). Lower panels show azimuthal averages at different time points. Scale bar, $5 \mu \mathrm{m}$. (b) Azimuthal average of fluorescence intensity inside condensates near the interface (magenta in (a)) for PGL-3 droplets at different salt concentrations (left, units: $\mathrm{mM}$ ) and two coacervate systems (right). (c) Flurorescence recovery inside a PGL-3 condensate, along the radial direction (azimuthal averages, see (a)). $D_{\text {in }}$ is extracted by global fitting of Eq. $1 \mathrm{~b}$ to the experimental profiles, using the experimentally extracted initial and boundary conditions (see panel (b)). The grey line indicates an offset that comes about due to incomplete bleaching, and a small but visible fast and uniform recovery with unknown cause (see Methods). (d) Comparison between $D_{\text {in }}$ and viscosity $\eta$ for PGL-3 condensates at different salt concentrations. Viscosity data taken from (Jawerth et al. (2018)) for untagged PGL-3. Note, GFP-tagged PGL-3 has a higher viscosity than untagged PGL-3, which means the hydrodynamic radius of PGL-3:GFP cannot be directly computed from this panel (Jawerth et al. (2020)). (e) Same as (c) but for a CMD/PLYS coacervate droplet. (f) Diffusion coefficients for coacervate systems. Each data point represents $D_{\text {in }}$ for a single droplet time course. Note the low spread of the measured values.

for untagged PGL-3 (Fig. 1 d). Using viscosity data for GFP-tagged PGL-3 (Jawerth et al. (2020)), we use the StokesSutherland-Einstein relationship $D_{\mathrm{in}}=k_{\mathrm{B}} T /(6 \pi a \eta)$ to estimate the hydrodynamic radius of PGL-3:GFP, $a=1.5 \mathrm{~nm}$ (Einstein (1905); Sutherland (1905); von Smoluchowski (1906)). This estimate is consistent with the value reported in

\section{Ref. Liarzi and Epel (2005).}

For the coacervate systems, we find for the diffusion coefficients inside $D_{\text {in }}=(1.68 \pm 0.09) \mu \mathrm{m}^{2} \mathrm{~s}^{-1}$ for PLYS/ATP coacervates and $D_{\text {in }}=(5.65 \pm 0.32) \mu \mathrm{m}^{2} \mathrm{~s}^{-1}$ for CMD/PLYS coacervates; see (Fig. 1f). The standard deviation of these measurements is low enough such that even a single measurement provides a good estimate of $D_{\text {in }}$. Interestingly, $D_{\text {in }}$ for the coacervate droplets is about 10 times smaller than the diffusion constant of the dilute polyelectrolytes, $D_{\text {out }}$ (Arrio-Dupont et al. (1996); Morga et al. (2019)).

\section{Theory for the dynamics of labeled molecules in phase-separating systems}

To understand the physical origin of the time-dependent concentration of unbleached components at the condensate interface and the phenomenological Eqs. (1) for the diffusion inside a condensate, we need a full theory for the diffusion inside, outside and across phase boundaries. Here, we derive such a theory for a system that can be described by a binary, incompressible mixture prior to photobleaching. This binary mixture is composed of condensate material and solvent. The condensate material has a concentration profile, $c_{\mathrm{tot}}(\boldsymbol{x}, t)=\phi_{\mathrm{tot}}(\boldsymbol{x}, t) / v$, which can be expressed in terms of a volume fraction profile $\phi_{\mathrm{tot}}(\boldsymbol{x}, t)$ by dividing through the molecular volume of the condensate material, $v$. Due to incompressibility, the solvent volume fraction in such a binary mixture is given by $\left(1-\phi_{\mathrm{tot}}\right)$. The system prior to photobleaching is assumed to be either at equilibrium, i.e., a single droplet and $\partial_{t} \phi_{\text {tot }}=0$, or close to equilibrium, i.e., a system composed of many 
a

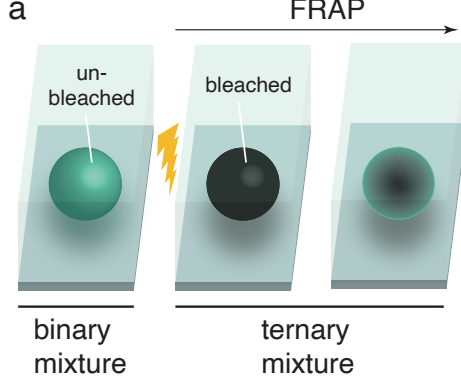

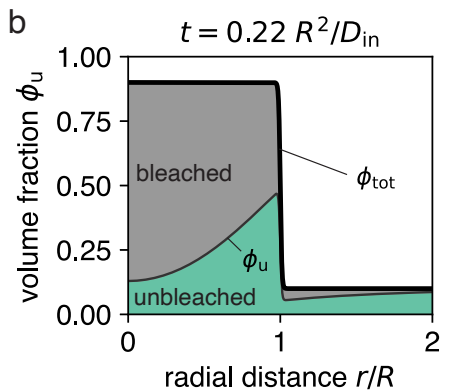

C

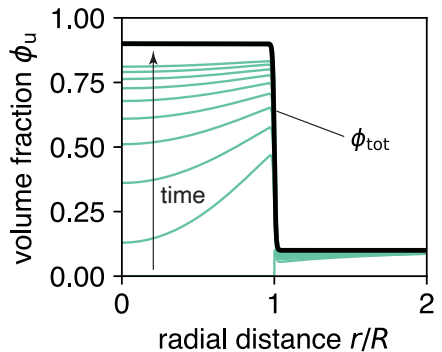

Figure 2. Ternary mixture accounts for the dynamics of bleached and unbleached molecules.

(a) Before bleaching, a droplet that is composed of fluorescently labelled molecules can be described by a binary mixture, namely unbleached molecules and solvent. After bleaching, the system is composed of three components, bleached molecules, unbleached molecules and solvent. If the system was at equilibrium prior to bleaching, the sum of bleached and unbleached molecules forms a stationary, non-uniform profile $\phi_{\text {tot }}(r)$ (see panel b). (b) Snapshot of model dynamics. Initial conditions are

$\phi_{\mathrm{u}}(r, t=0)=\phi_{\text {out }} \cdot \Theta(r-R)$, corresponding to a fully bleached droplet. Note that at any time we have $\phi_{\mathrm{tot}}=\phi_{\mathrm{u}}+\phi_{\mathrm{b}}$. (c) Time course of spatial recovery. For long times, when nearly all bleached material has been exchanged, $\phi_{\mathrm{u}}$ approaches $\phi_{\mathrm{tot}}$. Panels (c,d) use radial symmetry for illustration purposes, however, the theory is general (see Fig. 3).

droplets undergoing slow Ostwald ripening and fusion and $\partial_{t} \phi_{\text {tot }}(\boldsymbol{x}, t) \simeq 0$. Thus, the (quasi-) stationary profile $\phi_{\mathrm{tot}}(\boldsymbol{x})$ prescribes a physical constraint for FRAP dynamics.

After photobleaching, the system becomes a ternary incompressible mixture composed of bleached $(b)$ and unbleached (u) components, as well as solvent (Fig. 2a). Introducing the volume fraction of the bleached and unbleached components, $\phi_{b}$ and $\phi_{u}$, the physical constraint for the FRAP dynamics for a condensate at or close to equilibrium reads

$$
\phi_{\mathrm{tot}}(\boldsymbol{x})=\phi_{u}(\boldsymbol{x}, t)+\phi_{b}(\boldsymbol{x}, t)
$$

where the profiles of bleached and unbleached components depend on space and time during the FRAP dynamics, while $\phi_{\text {tot }}(\boldsymbol{x})$ depends on space only. Immediately after photobleaching, unbleached molecules diffuse into the condensate leading to FRAP dynamics of unbleached molecules inside (Fig. 2b,c). At long times, the concentration profile of unbleached molecules approaches the profile prior to photobleaching, $\phi_{\mathrm{tot}}(\boldsymbol{x})$. The dynamics of both concentration profiles, bleached and unbleached molecules, $c_{i}=\phi_{i} / v_{i}(i=b, u)$, with $v_{i}$ denoting the molecular volumes, is described by the following conservation laws $(j=u, b)$,

$$
\begin{aligned}
\partial_{t} c_{i} & =-\nabla \cdot \boldsymbol{j}_{i}, \\
\dot{j}_{i} & =-\Gamma_{i} \nabla \mu_{i}-\bar{\Gamma}_{i j} \nabla \mu_{j},
\end{aligned}
$$

Here, $\Gamma_{i}$ are the Onsager transport coefficients, often referred to as mobilities, and $\bar{\Gamma}_{i j}$ are the Onsager cross coupling coefficients obeying $\bar{\Gamma}_{i j}=\bar{\Gamma}_{j i}=: \bar{\Gamma}$. In general, both mobility coefficients depend on the volume fractions. To linear order, the flux $\boldsymbol{j}_{i}$ is driven by gradients in exchange chemical potentials $\mu_{i}$ and $\mu_{j}$. The exchange chemical potentials, $\mu_{i}=\delta F / \delta c_{i}$, are linked to the free energy, $F=\int d^{3} x f$, where $f$ denotes the free energy density. Expressing concentrations in terms of the volume fractions, $\phi_{i}=c_{i} v_{i}$, we describe our incompressible ternary mixture after photobleaching by a Flory-Huggins free energy density (Flory (1942); Huggins (1942); Krüger et al. (2018)):

$$
\begin{aligned}
f=\frac{k_{\mathrm{B}} T}{v_{\mathrm{sol}}} & {\left[\frac{\phi_{\mathrm{u}}}{n_{u}} \ln \phi_{\mathrm{u}}+\frac{\phi_{\mathrm{b}}}{n_{b}} \ln \phi_{\mathrm{b}}+\left(1-\phi_{\mathrm{u}}-\phi_{\mathrm{b}}\right) \ln \left(1-\phi_{\mathrm{u}}-\phi_{\mathrm{b}}\right)+\chi_{u s} \phi_{u}\left(1-\phi_{\mathrm{u}}-\phi_{\mathrm{b}}\right)+\chi_{b s} \phi_{b}\left(1-\phi_{\mathrm{u}}-\phi_{\mathrm{b}}\right)\right.} \\
+ & \left.\chi_{u b} \phi_{u} \phi_{b}+\frac{\kappa_{u}}{2}\left(\nabla \phi_{u}\right)^{2}+\frac{\kappa_{b}}{2}\left(\nabla \phi_{b}\right)^{2}+\frac{\kappa_{u b}}{2} \nabla \phi_{u} \cdot \nabla \phi_{b}\right],
\end{aligned}
$$

where we write the molecular volumes of bleached and unbleached components in $n_{i}$ multiples of the solvent molecular volume $v_{\text {sol }}$, i.e., $v_{i}=n_{i} v_{\text {sol }}$. Moreover, $\chi_{i j}$ denote dimensionless Flory-Huggins parameters characterizing the interactions between different components $i$ and $j$, where subscript $s$ indicates the solvent. The parameters $\kappa_{i}$ and $\kappa_{u b}$ characterize the free energy penalties for spatial heterogeneities and are linked to the surface tensions.

If photobleaching does not affect the molecular interactions or molecular volumes, the free energy density above can be simplified significantly (Appendix of Ref. (Krïger et al. (2018)). In this case, the interactions between unbleached and 
solvent, and bleached and solvent components are equal, $\chi_{u s}=\chi_{b s}=: \chi$, and cross interactions vanish to zero, $\chi_{u b}=0$. Moreover, molecular volumes of bleached and unbleached components are equal, $n_{u}=n_{b}=: n$, and the parameters characterizing free energy penalties for spatial heterogeneities obey $\kappa_{u}=\kappa_{b}=: \kappa$ and $\kappa_{u b}=2 \kappa$. Thus, the simplified free energy reads

$$
f=\frac{k_{\mathrm{B}} T}{v_{\mathrm{sol}}}\left[\frac{\phi_{\mathrm{u}}}{n} \ln \phi_{\mathrm{u}}+\frac{\phi_{\mathrm{b}}}{n} \ln \phi_{\mathrm{b}}+\left(1-\phi_{\mathrm{u}}-\phi_{\mathrm{b}}\right) \ln \left(1-\phi_{\mathrm{u}}-\phi_{\mathrm{b}}\right)+\chi\left(1-\phi_{\mathrm{u}}-\phi_{\mathrm{b}}\right)\left(\phi_{\mathrm{u}}+\phi_{\mathrm{b}}\right)+\frac{\kappa}{2}\left(\nabla\left(\phi_{\mathrm{u}}+\phi_{\mathrm{b}}\right)\right)^{2}\right] .
$$

To ensure a constant diffusion coefficient in the dilute limits of the bleached and unbleached components, we employ the scaling ansatz for a ternary mixture, $\Gamma_{i}=\Gamma_{0} \phi_{i}\left[\left(1-\phi_{\text {tot }}\right)+\left(\bar{\Gamma}_{0} / \Gamma_{0}\right) \phi_{j}\right]$ and $\bar{\Gamma}=-\bar{\Gamma}_{0} \phi_{u} \phi_{b}$. In general, both mobility functions, $\Gamma_{0}$ and $\bar{\Gamma}_{0}$, depend on the total volume fraction $\phi_{\text {tot }}$. For the limiting case where bleached and unbleached molecules are identical particles, we can choose $\Gamma_{0}=\bar{\Gamma}_{0}$. Applying the equilibrium FRAP condition (2) and using Eq. (3), we find that the concentration of unbleached components is governed by the following diffusion equation

$$
\partial_{\mathrm{t}} c_{u}=\nabla \cdot\left[D\left(\phi_{\mathrm{tot}}\right)\left(\nabla c_{u}-c_{u} \frac{\nabla \phi_{\mathrm{tot}}}{\phi_{\mathrm{tot}}}\right)\right],
$$

with a $\phi_{\text {tot }}(\boldsymbol{x})$-dependent diffusivity, $D\left(\phi_{\text {tot }}\right)=k_{\mathrm{B}} T \Gamma_{0}\left(\phi_{\mathrm{tot}}\right)$. As we show in Ref. (Bo et al. (2021)) a similar approach can be used to investigate single-molecule dynamics across phase boundaries.

Similar to Eq. (1), the diffusion equation above is linear in $c_{u}$. However, the dynamics of unbleached components are affected by gradients in $\phi_{\text {tot }}(x)$ and components diffuse with different diffusion coefficients inside and outside the condensate, where in each phase $\nabla \phi_{\text {tot }}=0$ (Fig. 2b). The position-dependence of $\phi_{\text {tot }}(x)$ is given by the equilibrium condition of a homogeneous chemical potential of the binary mixture prior to photobleaching, which implies $\partial_{t} \phi_{\text {tot }}=0$. For a radially symmetric system with $r$ denoting the radial coordinate, $\phi_{\text {tot }}(r)=\phi_{\text {out }}^{\text {eq }}+\left(\phi_{\text {in }}^{\text {eq }}-\phi_{\text {out }}^{\text {eq }}\right) H((r-R) / \ell)$, where $\phi_{\mathrm{in}}^{\mathrm{eq}}$ and $\phi_{\mathrm{out}}^{\mathrm{eq}}$ are the equilibrium volume fractions inside and outside, respectively, $R$ is the droplet radius, and $\ell$ denotes the width of the interface. Moreover, $H((r-R) / \ell)$ is a function that decreases from one to zero at $r=R$ on an interface width $\ell$. For phase separation close to the critical point and large droplet sizes, $H(x)=(1+\tanh (x)) / 2($ Bray (1994); Weber et al. (2019)). We numerically solve Eq. (6) using a finite element method (Logg et al. (2012)) in a finite domain of size $L$ which is much larger than the droplet radius $R$ and fit the solution to experimental data.

In summary, our model has seven parameters where four parameters, i.e., the equilibrium volume fractions $\phi_{\mathrm{in}}^{\mathrm{eq}}$ and $\phi_{\mathrm{in}}^{\mathrm{eq}}$, the interface width $\ell$, the droplet radius $R$, characterize the equilibrium profile prior to bleaching $\phi_{\text {tot }}(x)$. The remaining parameters are the system size $L$ and the diffusion coefficients inside and outside, which are given as

$$
\begin{gathered}
D_{\text {in }}=k_{\mathrm{B}} T \Gamma_{0}\left(\phi_{\text {in }}^{\mathrm{eq}}\right), \\
D_{\text {out }}=k_{\mathrm{B}} T \Gamma_{0}\left(\phi_{\text {out }}^{\mathrm{eq}}\right) .
\end{gathered}
$$

For the case of a single, spherical droplet with an infinitely thin interface (Weber et al. (2019)), we can derive an effective droplet model for the unbleached component from Eq. (6), where the dynamics of unbleached components inside and outside are given by diffusion equations that are coupled by boundary conditions (see App. 1 for the derivation):

$$
\begin{aligned}
\partial_{t} c_{u}(r, t) & =D_{\text {in }} \nabla^{2} c_{u}, \quad \text { for } r<R, \\
\partial_{t} c_{u}(r, t) & =D_{\text {out }} \nabla^{2} c_{u}, \quad \text { for } r>R, \\
-\left.D_{\text {in }} e_{r} \cdot \nabla c\right|_{R_{-}} & =-\left.D_{\text {out }} e_{r} \cdot \nabla c\right|_{R_{+}}, \\
c_{u}\left(r=R_{-}, t\right) & =P c_{u}\left(r=R_{+}, t\right) .
\end{aligned}
$$

Here, $\boldsymbol{R}_{-}$and $\boldsymbol{R}_{+}$denote the radial position directly inside and outside the droplet interface, respectively. Eq. (8c) describes an equality of the fluxes directly inside and outside of the interface, respectively, and thereby expresses particle number conservation at the interface $r=R$. Eq. (8d) describes a jump in concentration of unbleached components, which is determined by the thermodynamic partition coefficient

$$
P=\frac{\phi_{\text {in }}^{\mathrm{eq}}}{\phi_{\mathrm{out}}^{\mathrm{eq}}} .
$$

Moreover, the flux vanishes at the origin $r=0,\left.\boldsymbol{e}_{r} \cdot \nabla c\right|_{r=0}=0$, and at the system boundary $r=L,\left.\boldsymbol{e}_{r} \cdot \nabla c\right|_{r=L}=0$. Eqs. (8) were phenomenologically proposed in Ref. (Münchow et al. (2008)) to study protein diffusion across the interface 
in aqueous two-Phase systems. Moreover, Eqs. (8) were also studied in the context of FRAP of protein condensates in Ref. (Taylor et al. (2019)), where $D_{\text {in }}, D_{\text {out }}$ and $P$ were considered to be independent parameters. Strictly speaking, due to phase separation, the diffusion coefficients $D_{\text {in }}$ and $D_{\text {out }}$ are not independent which is evident in Eqs. (7). For example, in the absence of phase separation or at the critical point, $\phi_{\text {in }}^{\text {eq }}=\phi_{\text {out }}^{\text {eq }}$ (i.e., $P=1$ ), the diffusion coefficients inside and outside must be equal, $D_{\text {in }}=D_{\text {out }}$. Specifically, Eqs. (7) can be written as

$$
D_{\text {out }}(P)=D_{\text {in }} \frac{\Gamma_{0}\left(\phi_{\text {in }}^{\mathrm{eq}}\right)}{\Gamma_{0}\left(\phi_{\text {in }}^{\mathrm{eq}} / P\right)} .
$$

This expression indicates that for a given condensate with fixed $\phi_{\mathrm{in}}^{\mathrm{eq}}$ and $D_{\mathrm{in}}$, there is a relationship between the diffusivity outside $D_{\text {out }}$ and the partition coefficient $P$. However, except for the limit $P \rightarrow 1$, Eq. (10) does not impose further constraints on $D_{\text {out }}(P)$ due to the unknown mobility function $\Gamma_{0}\left(\phi_{\text {tot }}\right)$. For large $P$, the missing knowledge of the mobility function renders $D_{\text {out }}, D_{\text {in }}$ and $P$ as effectively independent parameters. This provides a theoretical justification for the phenomenological assumption given by (Taylor et al. (2019)), at least for large $P$.

In the following, we use our theory (Eq. (6)) to investigate the impact of the condensate environment on the FRAP dynamics. In particular, we consider how a passivated coverslip (no wetting of condensates) and nearby condensates affect the influx and thereby the recovery dynamics. Finally, we show how our theory can be used to determine the physical parameters corresponding to the condensate environment such as $D_{\text {out }}$ and $P$ from a single FRAP experiment.

\section{Impact of non-wetting coverslip on FRAP dynamics}

Here, we investigate the influence of the coverslip surface on the FRAP dynamics of non-wetting spherical droplets. Under typical in vitro conditions, condensates sediment due to gravity, leading to sessile droplets on a coverslip. In many experimental setups, coverslips are passivated, e.g. pegylated, in order to suppress wetting of condensates on the coverslip surface (Alberti et al. (2018)). These experimental conditions lead to almost spherical droplets since capillary effects are typically negligible for $\mu \mathrm{m}$-sized polymer-rich or protein-rich droplets (Park et al. (2013); McCall et al. (2020)).

We numerically solved Eq. (6) for a spherical condensate on top of a no-flux boundary that mimics the coverslip surface (Fig. 3a). We find that the recovery of the average volume fraction inside the condensate can slow down compared to the case without a coverslip (Fig. 3b). This slow-down vanishes if droplets have a distance to the coverslip surface larger than a few droplet diameters. Moreover, we find that the slow-down is more pronounced for larger partition coefficients $P$. This trend can be explained by the size of the region in the dilute phase from where most unbleached molecules are recruited: if $P$ is small, most of the unbleached molecules come from the immediate surroundings of the condensate. Hence the influence of radial asymmetry is minimal and the recovery appears almost unchanged compared to the case without coverslip. However, for large $P$, the condensate recruits unbleached molecules from distances far away, limiting the recruitment to an effective half-space compared with the case without coverslip. Indeed, for very large $P$ recovery rates slow down maximally by a factor of two.

Interestingly, by extracting the boundary concentration in mid-plane, similar to the procedure in Fig. 1, and spatially fitting the solutions of Eq. (1) to the ensuing recovery profiles, we can reliably recover the input diffusion coefficient $D_{\text {in }}$ (Fig. 3c). The reason for this excellent agreement is that by considering the intensity at the condensate interface, our method is independent of the time scale set by diffusion in the dilute phase.

\section{Impact of neighbouring condensates on FRAP dynamics}

In this section, we address the impact of neighbouring condensates on the recovery dynamics. We solved Eq. (6) for a system composed of a bleached condensate with four unbleached neighbouring condensates of the same size (Fig. 3d) and find that neighbouring condensates can significantly speed-up the recovery dynamics (Fig. 3e). This speed-up is only pronounced for rather close condensates with inter-droplet distance on the order of condensate size. Moreover, similar to the impact of the coverslip, the effects of the recovery dynamics are stronger for larger partition coefficients $P$. For small $P$, most unbleached molecules are recruited from the dilute phase leading to almost no effect on the recovery also when condensates are very close to each other (Fig. 3e, left). For large partition coefficients $P$, however, a certain fraction of molecules are recruited from the neighbouring condensates causing a significant speed-up of the recovery (Fig. 3e, right).

Again, despite this change in total recovery due to close-by neighbouring droplets, we can reliably measure $D_{\text {in }}$ via our spatial fitting method (Fig. 3f). In particular, by extracting the boundary intensity in mid-plane and spatially fitting the solutions of Eq. (1), we find very good agreement with our input $D_{\text {in }}$. This agreement shows that our method is robust for typical experimental systems that deviate from an ideal isolated condensate. 
bioRxiv preprint doi: https://doi.org/10.1101/2021.03.08.434288; this version posted March 8, 2021. The copyright holder for this preprint (which was not certified by peer review) is the author/funder, who has granted bioRxiv a license to display the preprint in perpetuity. It is made available under aCC-BY-NC 4.0 International license.

a

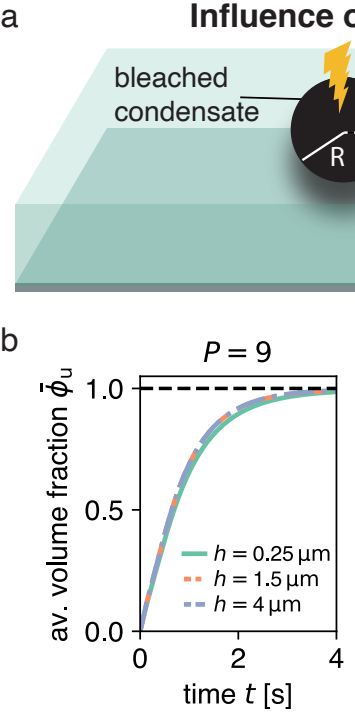

C

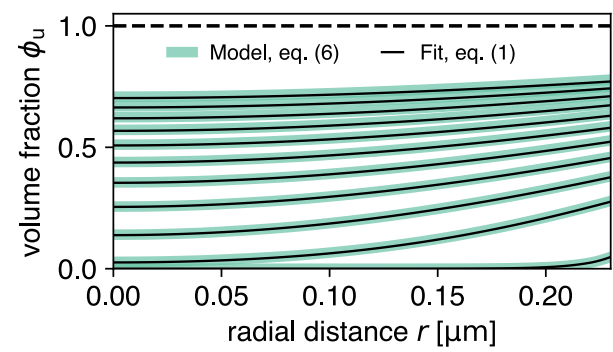

d Influence of droplet neighbours

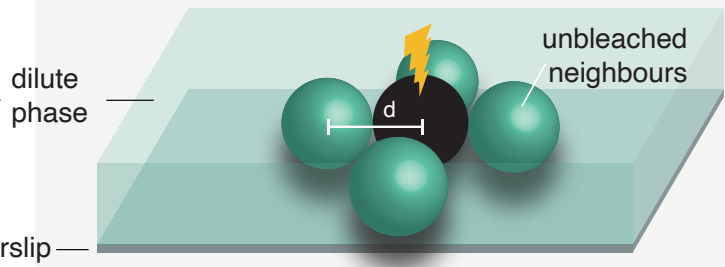

e
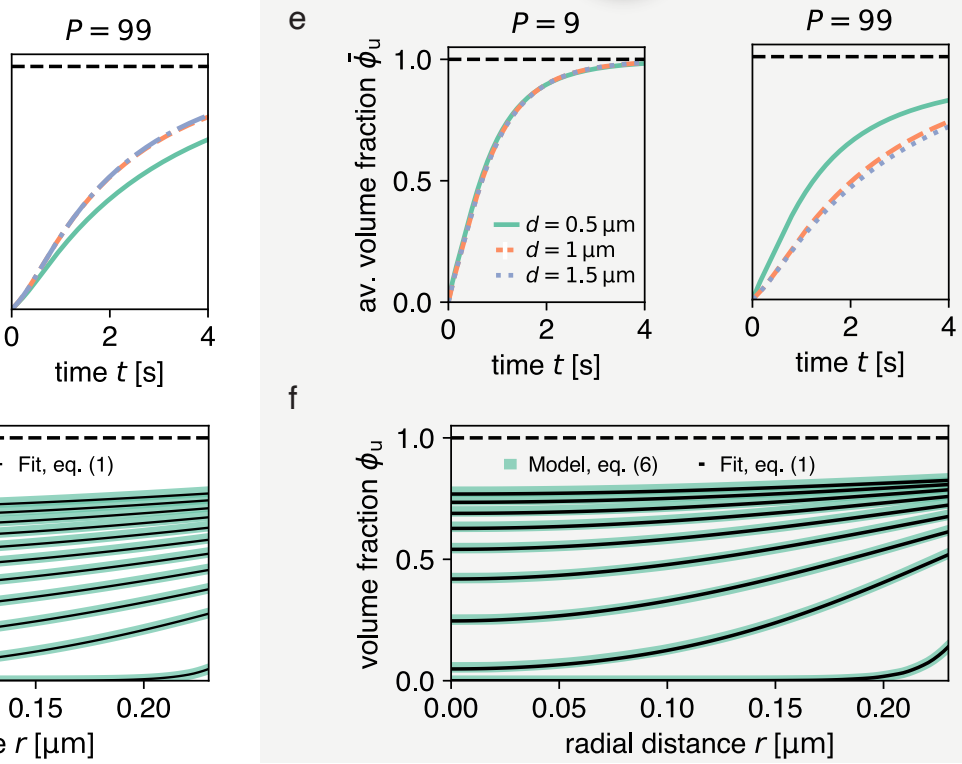

Figure 3. Impact of droplet environment on recovery dynamics.

(a) Sketch of a typical experimental set-up with a droplet above a passivated coverslip, where the droplet center has a distance $h>R$ to the coverslip. (b) Recovery of average unbleached volume fraction $\bar{\phi}_{\mathrm{u}}(t)=\int d^{3} r \phi_{\mathrm{u}}(\vec{r}, t) \cdot 3 /\left(4 \pi R^{3}\right)$ for different heights $h$ above the coverslip at different partition coefficients $P$. Results were obtained from finite element studies of Eq. (6) considering the geometries depicted in (a). For even larger $h$-values (e.g., no coverslip), results are approximately equal to the blue dashed line. (c) Using the method introduced in Fig. 1 on the worst case scenario $h=0.25 \mu \mathrm{m}$ in (a) results in an excellent fit and can reliably extract the input $D_{\text {in }}$. (d) Sketch of neighbouring droplets next to a bleached droplet. (e) Total recovery curves for finite element simulations of the geometry depicted in (d), for different distances between neighbouring droplet centers, at different partition coefficients $P$. Note the strong dependence on the distance of neighbouring droplets. For even larger $d$-values (e.g., no neighbouring drops), results are approximately equal to the blue dashed line. (f) Same as (c) but for worst case of (e), i.e. $d=0.5 \mu \mathrm{m}$. 

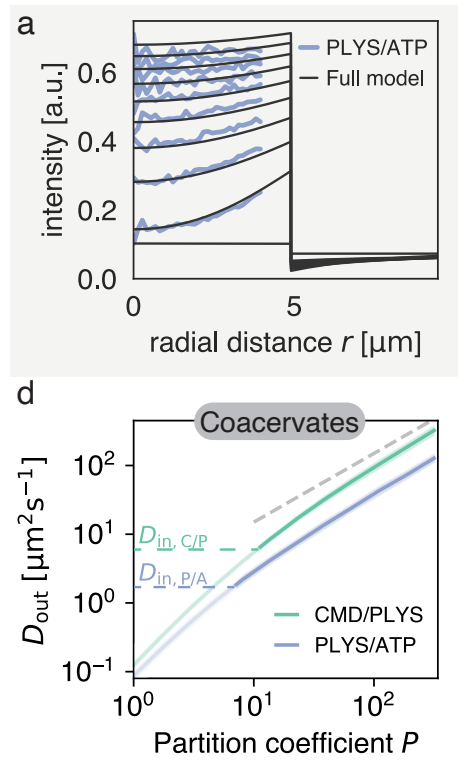
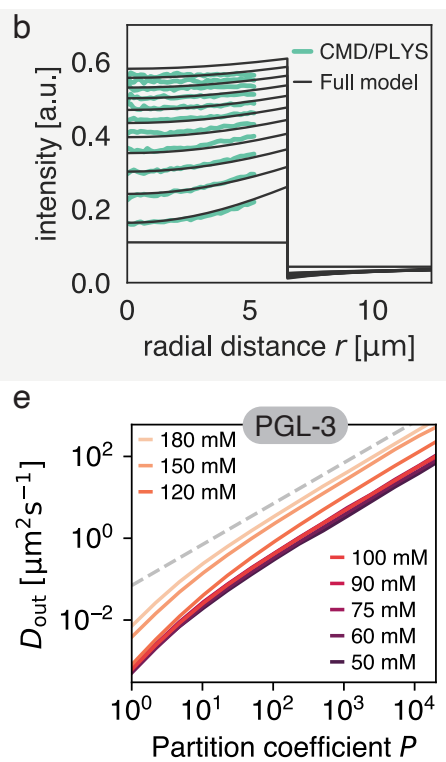

C

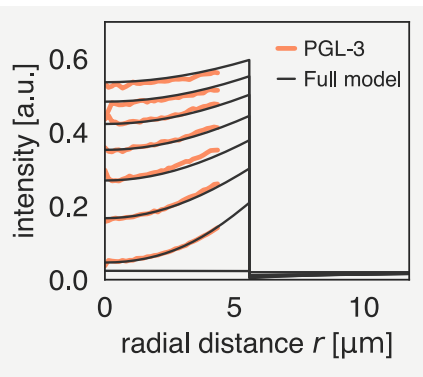

f

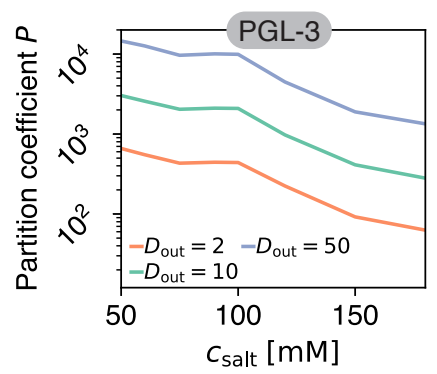

Figure 4. Varying partition coefficient $P$ and diffusivity outside $D_{\text {out }}$ simultaneously can lead to similar recovery kinetics. (a) Spatial recovery of a single PLYS/ATP droplet (blue) with a fit to the full model (black). Note, data close to the droplet boundary cannot be fit, due to optical artefacts giving rise to an artificially broad interface (see methods and Fig. 1a). (b) Same as (a) for a CMD/PLYS coacervate droplet. (c) Same as (a) for a PGL-3 droplet. (d) Given the partition coefficient $P, D_{\text {out }}$ is found by fitting the coacervate data to the model. Note the convergence to a power law, $D_{\text {out }} \propto P^{n}$ with $n=1$ for large partition coefficients (grey dashed line; for a discussion see Appendix 2). Average $D_{\text {in }}$ values as obtained in Fig. If are indicated for both systems. Values with $D_{\text {out }}<D_{\text {in }}$ are light shaded. (e) Same as (b) but for PGL-3 with different salt concentrations. Note the order from top to bottom from highest to lowest salt concentration. (f) Based on (e), partition coefficients $P\left(c_{\text {salt }}\right)$ can be estimated for a given $D_{\text {out }}$. $P\left(c_{\text {salt }}\right)$ is shown for different $D_{\text {out }}$. Units of $D_{\text {out }}$ in $\mu \mathrm{m}^{2} \mathrm{~s}^{-1}$.

\section{How to determine partition coefficients and outside diffusivity via FRAP of condensates}

We have shown that by using the time-dependent fluorescence at the interface of a spherical droplet we can accurately fit our dynamic Eq. (1) to our experimental data and thus determine the diffusion constant inside the droplet, $D_{\text {in }}$ (Fig. 1a). Our theory (see Eq. 6) suggests that the fluorescence at the droplet interface is affected by the physical parameters characterizing the droplet environment such as the diffusion coefficient $D_{\text {out }}$ and the partition coefficient $P$. In particular, the flux through the droplet interface is enlarged for increasing $D_{\text {out }}$ or decreasing $P$ (see Eq. (8c) after rescaling the concentration close to interface). Thus, for a condensate with concentration $\phi_{\text {in }}^{\text {eq }}$ and diffusion coefficient $D_{\text {in }}$, the flux between both phases at the interface implies a relationship between $D_{\text {out }}$ and $P$, as suggested by Eq. (10). However, due to the unknown mobility function, we need to obtain this relationship from experimental data.

Here, we determined the relationship between $D_{\text {out }}$ and $P$ by fitting numerical solutions of Eq. (6) to the recovery dynamics inside the droplet (Fig. 4a-c and supp. movies 1,2,3). The diffusion coefficient inside, $D_{\text {in }}$, was independently determined for each experiment via our method introduced in Fig. 1a. This leaves $P$ and $D_{\text {out }}$ as independent parameters, which is valid for large $P$ (see discussion after Eq. (10)). We thus sampled $P$ (with $P \gg 1$ ) across three orders of magnitude and obtained the best-fitting $D_{\text {out }}$ for each $P$ (Fig. 4d,e). Notably, all the combinations of $D_{\text {out }}$ and $P$ represent relatively good fits. For very large $P$, we find that the best $D_{\text {out }}$ scales linearly with $P$ (dashed lines in Fig. 4d,e); for a discussion on the origin of this scaling, please refer to Appendix 2.

Strikingly, each experimental condition leads to a unique relationship between $D_{\text {out }}$ and $P$. This relationship allows one to obtain the partition coefficient $P$ by measuring $D_{\text {out }}$, e.g. by using FCS. We can also use this relationship to obtain insight into how the addition of salt affects the partition coefficient of PGL-3. The outside diffusivity $D_{\text {out }}$ can be estimated based on the value for diffusion of eGFP in water, $D_{\text {out,GFP }}=87 \mu \mathrm{m}^{2} \mathrm{~s}^{-1}$ (Arrio-Dupont et al. (2000)). eGFP consists of 239 amino acids, while PGL-3 consists of 693 amino acids. If we assume the tagged protein to be spherical and use the Stokes-Sutherland-Einstein relationship $D \propto 1 / a$, we obtain $D_{\text {out }} \approx 44 \mu \mathrm{m}^{2} \mathrm{~s}^{-1}$. Specifically, for salt concentrations in the range from $50 \mathrm{mM}$ to $180 \mathrm{mM}$, the partition coefficient $P$ of PGL-3 droplets decreases more than 10-fold (Fig. 4f). This trend is probably a result of enhanced screening of charged groups for increasing salt concentration 
a

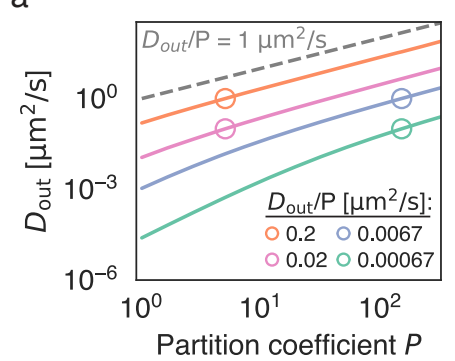

b

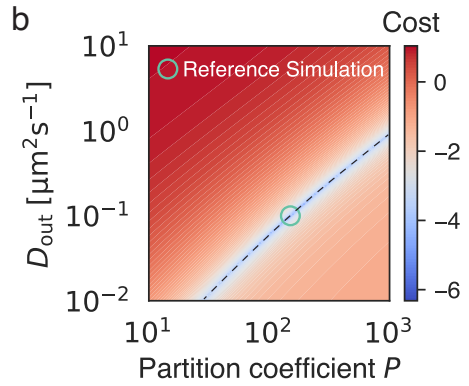

c

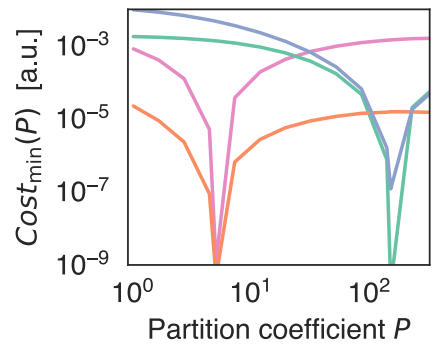

Figure 5. Given the recovery dynamics inside a condensate, the key parameters $D_{\text {in }}, D_{\text {out }}$ and $P$ can be determined uniquely without measuring the outside dynamics.

As reference systems, indicated by open circles, we consider in silico data, obtained by solving Eq. (6) with known parameters $P$ and $D_{\text {out }}$. To mimic the approach of initially determining $D_{\text {in }}$ (see Fig. 1) we keep $D_{i n}=0.01 \mu \mathrm{m}^{2} \mathrm{~s}^{-1}$ for all in silico datasets. (a) Given the partition coefficient $P, D_{\text {out }}$ is found by fitting synthetically generated data to the model. (b) Cost function (colorbar, log-scale) as a function of $D_{\text {out }}$ and $P$. We note that the minimum at the reference simulations coincides with the parameters used to generate the synthetic data. The valley in parameter space (dashed line) corresponds to the green line in (a). (c) Minimum of cost function for each $P$, corresponding to curves shown in (a). This minimum corresponds to the valley indicated by the dashed line in (b). Note the minimum at the input parameter set, which indicates uniqueness of the outside dynamics for given values of $D_{\text {out }}$ and $P$.

and is in line with unpublished results using a technique relying on the measurement of droplet volumes (A.W. Fritsch and J.M. Iglesias-Artola, personal communication). To obtain more precise estimates of $P$, Figs. $4 \mathrm{~d}$,e can be complemented by additional measurements of $D_{\text {out }}$. Note, the absolute values of $P$ are overestimated up to twofold, since the fitting method employed here assumes spherical symmetry, thus effectively ignoring the effect of the coverslip (see caption of Fig. 3 for a discussion).

We next asked, whether there is a way to bypass this extra measurement and obtain specific values for $P$ and $D_{\text {out }}$ at the same time, without measuring fluorescence intensities outside the droplet. Though each combination $\left(D_{\text {out }}, P\right)$ along the lines specified in Figs. 4d,e leads to a reasonable fit, we will now show that there is a distinct combination that exhibits a global minimum of the cost function for each $D_{\text {out }}(P)$. Providing experimental evidence of this global minimum can be hampered by environmental effects such as neighbouring droplets or the coverslip surface (Fig. 3a,d). In particular, in our experimental studies, interdroplet distances are sometimes on the order of the droplet size and diffusive exchange is affected by the coverslip. Thus, we decided to use our model to create in silico data and provide evidence for the existence of a distinct combination of $D_{\text {out }}$ and $P$ for a fixed $D_{i n}$. Fixing $D_{\text {in }}$ mimics the approach of initially determining $D_{\text {in }}$, as outlined in Fig. 1. To determine the relationship $D_{\text {out }}(P)$, we proceed as described for Fig. 4. Fig. 5(a) depicts the $D_{\text {out }}(P)$ relationships corresponding to four parameter combinations in a range relevant for protein condensates and coacervate droplets. In particular, we choose two outside diffusivities $D_{\text {out }}$ of $0.1 \mu \mathrm{m}^{2} \mathrm{~s}^{-1}$ and $1 \mu \mathrm{m}^{2} \mathrm{~s}^{-1}$ and two partition coefficients, $P=5$ and $P=150$. We find indeed that each cost function exhibits a unique minimum for each of the considered parameter combinations (Fig. 5(b,c)). These findings indicate that all three parameters, $D_{\text {in }}, D_{\text {out }}$ and $P$, can in principle be determined by a single FRAP experiment of the droplet inside. Thus, in principle, there is no need to measure kinetic properties of the dilute phase to fully characterise the system in terms of its parameters. This possibility represents a new approach to determine the partition coefficient $P$, which is particularly important in light of recent data showing that measurements based on fluorescence intensity can lead to drastic underestimation of $P$ (McCall et al. (2020)).

\section{Discussion}

The dynamic redistribution of fluorescent molecules has been used to characterize liquid phase separation in biology via a variety of techniques, including SPT, FRAP and FCS (Elbaum-Garfinkle et al. (2015); Taylor et al. (2019); Moon et al. (2019)). Here, we have derived a theory from first principles that describes the diffusive motion of labeled molecules based on the physics of phase separation. It can be applied to many state-of-the art fluorescent methods such as FCS, SPT and FRAP and will thus be crucial help extend traditional techniques to the realms of phase separation ( (Ries and Schwille (2012))). Importantly, this theory enables us to avoid commonly applied approximations such as the frequently used single-exponential recovery (Brangwynne et al. (2009); Frottin et al. (2019); Kaur et al. (2019); Fisher and Elbaum-Garfinkle (2020); Kistler et al. (2018)).

Our theory describes the dynamics of labeled molecules through interfaces of condensates. It can be applied to 
spherical condensates but also non-spherical condensates and arbitrary bleach geometries, see Fig. 3. We were able to quantify the impact of neighbouring droplets and the coverslip on the recovery dynamics. We found that neighbouring droplets caused an appreciable speed-up in overall recovery, while emulating a coverslip caused a weak slow-down. In order to experimentally verify our theory, we have used three in vitro droplet systems, two composed of charged synthetic polymers and one with a purified protein component. There is remarkable quantitative agreement between our theory and the diffusion dynamics observed inside such droplets. This agreement shows that proteins and charged, synthetic polymers form droplets that follow simple diffusive dynamics inside. Crucially, we use the full spatio-temporal data for fitting and can thus distinguish the timescale set by intra-droplet diffusion from the timescales at play in the dilute phase. We extract the intensity directly at the inside of the droplet interface and fit a spatially resolved diffusion equation to the ensuing recovery. We use the boundary intensity as a dynamic boundary condition and the experimentally measured profile as initial condition. Throughout the time course, we find excellent agreement with the data and have thus found a method with minimal approximations that can precisely measure the inside diffusion coefficient $D_{\text {in }}$.

Building on the analysis of the droplet phase, we show that there is a relationship between partition coefficient $P$ and the outside diffusion coefficient $D_{\text {out }}$. Data obtained from FRAP experiments define a line in $\left(D_{\text {out }}, P\right)$ space, along which any parameter set can reliably account for the boundary dynamics. This unique relationship allows measuring $P$, when $D_{\text {out }}$ is known, and vice versa, which opens an interesting avenue for measuring partition coefficients purely based on dynamics. This is particularly important in light of recent data obtained by quantitative phase microscopy (QPM), which show that measuring partition coefficients based on fluorescence intensity can lead to strong underestimation of $P$ (McCall et al. (2020)). In the future, it would thus be interesting to use FCS to determine $D_{\text {out }}$ and compare the partition $P$ inferred by our method with measurements by QPM.

Our approach can be readily extended to multi-component systems with an arbitrary number of components, which is particularly useful in vivo. This would be hardly possible for techniques that do not use labeled components, such as QPM or other scattering methods. Of particular interest are multi-component systems with chemical reactions away from equilibrium. Our approach can then be used to determine the diffusion coefficients and concentration levels of reactants, and thereby provide insights into reaction kinetics. Interestingly, introducing the bleached molecules via a ternary mixture also enabled us to derive the Langevin equation governing single-molecule motion in phase-separated media, thus providing a link to SPT (Bo et al. (2021), in preparation). Approaches for single labelled molecules are highly relevant since high labeling fractions were shown to alter the kinetics in dense protein phases (Jawerth et al. (2018, 2020)). Finally, our techniques can also be employed to characterize rheological properties of condensates such as the recently reported glass-like dynamics of protein droplets (Jawerth et al. (2020)).

\section{Acknowledgements}

We are grateful to P. McCall, A. Fritsch, J.M. Iglesias-Artola, G. Bartolucci, T. Wiegand, M. Karnat, E. Filippidi, T. Harmon, F. Jülicher and members of the Weber and Hyman groups for stimulating discussions, and P. McCall and J. Pfanzelter for very valuable and insightful comments on the manuscript. L. Hubatsch and C. Weber acknowledge the SPP 2191 "Molecular Mechanisms of Functional Phase Separation" of the German Science Foundation for financial support.

\section{Supplementary}

\section{Movie S1: FRAP dynamics in a PGL-3 droplet}

Left: Representative in vitro droplet after full bleach. Time course starts after a small time lag due to a fast uniform recovery (see methods).

Middle: Diffusion eq. (1) fit to azimuthally averaged droplet intensity with a global fit parameter $D_{\text {in }}$.

Right: Full model eq. (6) fit to azimuthally averaged droplet intensity.

\section{Movie S2: FRAP dynamics in a CMD/PLYS coacervate}

For description see Movie S1.

Movie S3: FRAP dynamics in a PLYS/ATP coacervate

For description see Movie S1. 
bioRxiv preprint doi: https://doi.org/10.1101/2021.03.08.434288; this version posted March 8, 2021. The copyright holder for this preprint (which was not certified by peer review) is the author/funder, who has granted bioRxiv a license to display the preprint in perpetuity. It is made available under aCC-BY-NC 4.0 International license.

\section{References}

Alberti S, Saha S, Woodruff JB, Franzmann TM, Wang J, Hyman AA. A User's Guide for Phase Separation Assays with Purified Proteins. Journal of Molecular Biology. 2018; 430(23):4806-4820. https://doi.org/10.1016/j.jmb.2018.06.038, doi: 10.1016/j.jmb.2018.06.038.

Arrio-Dupont M, Cribier S, Foucault G, Devaux PF, D’Albis A. Diffusion of fluorescently labeled macromolecules in cultured muscle cells. Biophysical Journal. 1996; 70(5):2327-2332. http://dx.doi.org/10.1016/S0006-3495(96)79798-9, doi: 10.1016/S00063495(96)79798-9.

Arrio-Dupont M, Foucault G, Vacher M, Devaux PF, Cribier S. Translational diffusion of globular proteins in the cytoplasm of cultured muscle cells. Biophysical Journal. 2000; 78(2):901-907. http://dx.doi.org/10.1016/S0006-3495(00)76647-1, doi: 10.1016/S0006-3495(00)76647-1.

Banani SF, Lee HO, Hyman AA, Rosen MK. Biomolecular condensates: Organizers of cellular biochemistry. Nature Reviews Molecular Cell Biology. 2017; 18(5):285-298. doi: 10.1038/nrm.2017.7.

Banerjee PR, Milin AN, Moosa MM, Onuchic PL, Deniz AA. Reentrant Phase Transition Drives Dynamic Substructure Formation in Ribonucleoprotein Droplets. Angewandte Chemie International Edition. 2017; 56(38):11354-11359. https: //onlinelibrary.wiley. com/doi/abs/10.1002/anie.201703191, doi: https://doi.org/10.1002/anie.201703191.

Bo S, Hubatsch L, Bauermann J, Weber CA, Jülicher F. Stochastic dynamics of single molecules across phase boundaries. unpublished. $2021 ;$.

Brangwynne CP, Eckmann CR, Courson DS, Rybarska A, Hoege C, Gharakhani J, Julicher F, Hyman AA. Germline P granules are liquid droplets that localize by controlled dissolution/condensation. Science. 2009; 324(5935):1729-1732. http://www.ncbi.nlm. nih.gov/pubmed/19460965http://www.sciencemag.org/content/324/5935/1729.full.pdf, doi: 10.1126/science.1172046.

Bray AJ. Theory of phase-ordering kinetics. Advances in Physics. 1994; 43(3):357-459. doi: 10.1080/00018739400101505.

Diaspro A. Optical fluorescence microscopy: From the spectral to the nano dimension. Springer Science \& Business Media; 2010.

Einstein A. Über die von der molekularkinetischen Theorie der Wärme geforderte Bewegung von in ruhenden Flüssigkeiten suspendierten Teilchen. Annalen der Physik. 1905; 322(8):549-560. http://dx.doi.org/10.1002/andp.19053220806, doi: 10.1002/andp.19053220806.

Elbaum-Garfinkle S, Kim Y, Szczepaniak K, Chen CCH, Eckmann CR, Myong S, Brangwynne CP. The disordered P granule protein LAF-1 drives phase separation into droplets with tunable viscosity and dynamics. Proceedings of the National Academy of Sciences. 2015; 112(23):7189-7194. https://www.pnas.org/content/112/23/7189, doi: 10.1073/pnas.1504822112.

Elson EL. Fluorescence correlation spectroscopy measures molecular transport in cells. Traffic. 2001; 2(11):789-796. doi: 10.1034/j.1600-0854.2001.21107.x.

Fisher RS, Elbaum-Garfinkle S. Tunable multiphase dynamics of arginine and lysine liquid condensates. Nature Communications. 2020; 11(1). doi: 10.1038/s41467-020-18224-y.

Flory PJ. Thermodynamics of High Polymer Solutions. The Journal of Chemical Physics. 1942 jan; 10(1):51-61. https://doi.org/10. 1063/1.1723621, doi: 10.1063/1.1723621.

Frottin F, Schueder F, Tiwary S, Gupta R, Körner R, Schlichthaerle T, Cox J, Jungmann R, Hartl FU, Hipp MS. The nucleolus functions as a phase-separated protein quality control compartment. Science. 2019; 365(6451):342-347. doi: 10.1126/science.aaw9157.

Goehring NW, Chowdhury D, Hyman AA, Grill SW. FRAP analysis of membrane-associated proteins: lateral diffusion and membranecytoplasmic exchange. Biophys J. 2010; 99(8):2443-2452. http://www.ncbi.nlm.nih.gov/pubmed/20959084http://ac.els-cdn. com/S0006349510010283/1-s2.0-S0006349510010283-main.pdf? tid=2a6fdf88-a8b9-11e5-90b4-00000aacb361\& acdnat=1450795073_2194f9adc3352ef913995fe24a7c93c7, doi: 10.1016/j.bpj.2010.08.033.

Griffin EE. Cytoplasmic localization and asymmetric division in the early embryo of Caenorhabditis elegans. Wiley Interdiscip Rev Dev Biol. 2015; 4(3):267-282. http://www.ncbi.nlm.nih.gov/pubmed/25764455http://onlinelibrary.wiley.com/store/10.1002/ wdev.177/asset/wdev177.pdf?v=1\&t=iihhsbds\&s=5b549a8fd9bcc4a249c07828f77d8342bcfda787, doi: 10.1002/wdev.177.

Guillén-Boixet J, Kopach A, Holehouse AS, Wittmann S, Jahnel M, Schlüßler R, Kim K, Trussina IREA, Wang J, Mateju D, Poser I, Maharana S, Ruer-Gruß M, Richter D, Zhang X, Chang YT, Guck J, Honigmann A, Mahamid J, Hyman AA, et al. RNA-Induced Conformational Switching and Clustering of G3BP Drive Stress Granule Assembly by Condensation. Cell. 2020; 181(2):346-361.e17. https://www.sciencedirect.com/science/article/pii/S0092867420303421, doi: https://doi.org/10.1016/j.cell.2020.03.049. 
Hubstenberger A, Noble SL, Cameron C, Evans TC. Translation Repressors, an RNA Helicase, and Developmental Cues Control RNP Phase Transitions during Early Development. Developmental Cell. 2013; 27(2):161-173. https://www.sciencedirect.com/ science/article/pii/S1534580713005728, doi: https://doi.org/10.1016/j.devce1.2013.09.024.

Huggins ML. Some properties of solutions of long-chain compounds. The Journal of Physical Chemistry. 1942; 46(1):151-158.

Hyman AA, Weber CA, Jülicher F. Liquid-Liquid Phase Separation in Biology. Annual Review of Cell and Developmental Biology. 2014 oct; 30(1):39-58. http://www. annualreviews.org/doi/10.1146/annurev-cellbio-100913-013325, doi: 10.1146/annurevcellbio-100913-013325.

Jawerth L, Fischer-Friedrich E, Saha S, Wang J, Ijavi M, Saha S, Hyman AA, Jülicher F. Protein condensates as aging Maxwell fluids. Science. 2020; (December)

Jawerth LM, Ijavi M, Ruer M, Saha S, Jahnel M, Hyman AA, Jülicher F, Fischer-Friedrich E. Salt-Dependent Rheology and Surface Tension of Protein Condensates Using Optical Traps. Physical Review Letters. 2018; 121(25). doi: 10.1103/PhysRevLett.121.258101.

Kaur T, Alshareedah I, Wang W, Ngo J, Moosa MM, Banerjee PR. Molecular crowding tunes material states of ribonucleoprotein condensates. Biomolecules. 2019; 9(2):1-17. doi: 10.3390/biom9020071.

Kistler KE, Trcek T, Hurd TR, Chen R, Liang FX, Sall J, Kato M, Lehmann R. Phase transitioned nuclear oskar promotes cell division of drosophila primordial germ cells. eLife. 2018; 7:1-35. doi: 10.7554/eLife.37949.

Krüger S, Weber CA, Sommer JU, Jülicher F. Discontinuous switching of position of two coexisting phases. New Journal of Physics. 2018 jul; 20(7):75009. https://doi.org/10.1088\%2F1367-2630\%2Faad173, doi: 10.1088/1367-2630/aad173.

Liarzi O, Epel BL. Development of a quantitative tool for measuring changes in the coefficient of conductivity of plasmodesmata induced by developmental, biotic, and abiotic signals. Protoplasma. 2005; 225(1):67-76. https://doi.org/10.1007/s00709-004-0079-x, doi: 10.1007/s00709-004-0079-x.

Logg A, Mardal KA, Wells GN, Others. Automated Solution of Differential Equations by the Finite Element Method. Springer; 2012. doi: 10.1007/978-3-642-23099-8.

Lyon AS, Peeples WB, Rosen MK. A framework for understanding the functions of biomolecular condensates across scales. Nature Reviews Molecular Cell Biology. 2020; http://dx.doi.org/10.1038/s41580-020-00303-z, doi: 10.1038/s41580-020-00303-z.

McCall PM, Kim K, Fritsch AW, Iglesias-Artola JM, Jawerth LM, Wang J, Ruer M, Peychl J, Poznyakovskiy A, Guck J, Alberti S, Hyman AA, Brugués J. Quantitative phase microscopy enables precise and efficient determination of biomolecular condensate composition. bioRxiv. 2020; p. 2020.10.25.352823. https://doi.org/10.1101/2020.10.25.352823.

McCall PM, MacKintosh FC, Kovar DR, Gardel ML. Cofilin drives rapid turnover and fluidization of entangled F-actin. Proceedings of the National Academy of Sciences of the United States of America. 2019; 116(26):12629-12637. doi: 10.1073/pnas.1818808116.

Mir M, Bickmore W, Furlong EEM, Narlikar G. Chromatin topology, condensates and gene regulation: shifting paradigms or just a phase? Development (Cambridge, England). 2019; 146(19):1-6. http://www.ncbi.nlm.nih.gov/pubmed/31554625, doi: 10.1242/dev.182766.

Moon SL, Morisaki T, Khong A, Lyon K, Parker R, Stasevich TJ. Multicolour single-molecule tracking of mRNA interactions with RNP granules. Nature Cell Biology. 2019; 21(2):162-168. http://dx.doi.org/10.1038/s41556-018-0263-4, doi: 10.1038/s41556018-0263-4.

Morga M, Adamczyk Z, Kosior D, Kujda-Kruk M. Kinetics of Poly- 1 -lysine Adsorption on Mica and Stability of Formed Monolayers: Theoretical and Experimental Studies. Langmuir. 2019; 35(37):12042-12052. doi: 10.1021/acs.langmuir.9b02149.

Münchow G, Schönfeld F, Hardt S, Graf K. Protein diffusion across the interface in aqueous two-phase systems. Langmuir. 2008; 24(16):8547-8553.

Park J, Park J, Lim H, Kim HY. Shape of a large drop on a rough hydrophobic surface. Physics of Fluids. 2013; 25(2):022102.

Patel A, Lee HO, Jawerth L, Maharana S, Jahnel M, Hein MY, Stoynov S, Mahamid J, Saha S, Franzmann TM, Pozniakovski A, Poser I, Maghelli N, Royer LA, Weigert M, Myers EW, Grill S, Drechsel D, Hyman AA, Alberti S. A Liquid-to-Solid Phase Transition of the ALS Protein FUS Accelerated by Disease Mutation. Cell. 2015; 162(5):1066-1077. http://dx.doi.org/10.1016/j.cell.2015.07.047, doi: 10.1016/j.cell.2015.07.047.

Ries J, Schwille P. Fluorescence correlation spectroscopy. BioEssays. 2012; 34(5):361-368.

Rigler R, Elson ES. Fluorescence correlation spectroscopy: theory and applications, vol. 65. Springer Science \& Business Media; 2012. 
Robin FB, McFadden WM, Yao B, Munro EM. Single-molecule analysis of cell surface dynamics in Caenorhabditis elegans embryos. Nat Methods. 2014; 11(6):677-682. http://www.ncbi.nlm.nih.gov/pubmed/24727651http://www. nature.com/nmeth/ journal/v11/n6/pdf/nmeth.2928.pdf, doi: 10.1038/nmeth.2928.

Saha S, Weber CA, Nousch M, Adame-Arana O, Hoege C, Hein MY, Osborne-Nishimura E, Mahamid J, Jahnel M, Jawerth L, Pozniakovski A, Eckmann CR, Jülicher F, Hyman AA. Polar Positioning of Phase-Separated Liquid Compartments in Cells Regulated by an mRNA Competition Mechanism. Cell. 2016; 166(6):1572-1584.e16. doi: 10.1016/j.cell.2016.08.006.

Sanders DW, Kedersha N, Lee DSW, Strom AR, Drake V, Riback JA, Bracha D, Eeftens JM, Iwanicki A, Wang A, Wei MT, Whitney G, Lyons SM, Anderson P, Jacobs WM, Ivanov P, Brangwynne CP. Competing Protein-RNA Interaction Networks Control Multiphase Intracellular Organization. Cell. 2020; 181(2):306-324.e28. https://www.sciencedirect.com/science/article/pii/ S0092867420303433, doi: https://doi.org/10.1016/j.cell.2020.03.050.

Saxton MJ, Jacobson K. Single-particle tracking: applications to membrane dynamics. Annual review of biophysics and biomolecular structure. 1997; 26(1):373-399.

von Smoluchowski M. Zur kinetischen Theorie der Brownschen Molekularbewegung und der Suspensionen. Annalen der Physik. 1906 jan; 326(14):756-780. https://doi.org/10.1002/andp.19063261405, doi: https://doi.org/10.1002/andp.19063261405.

Sprague BL, Pego RL, Stavreva DA, McNally JG. Analysis of binding reactions by fluorescence recovery after photobleaching. Biophysical Journal. 2004; 86(6):3473-3495. http://dx.doi.org/10.1529/biophysj.103.026765, doi: 10.1529/biophysj.103.026765.

Stasevich TJ, Mueller F, Michelman-Ribeiro A, Rosales T, Knutson JR, McNally JG. Cross-validating FRAP and FCS to quantify the impact of photobleaching on in vivo binding estimates. Biophysical Journal. 2010; 99(9):3093-3101. http://dx.doi.org/10.1016/j. bpj.2010.08.059, doi: 10.1016/j.bpj.2010.08.059.

Sutherland W. LXXV. A dynamical theory of diffusion for non-electrolytes and the molecular mass of albumin. The London, Edinburgh, and Dublin Philosophical Magazine and Journal of Science. 1905 jun; 9(54):781-785. https://doi.org/10.1080/ 14786440509463331, doi: 10.1080/14786440509463331.

Taylor NO, Wei MT, Stone HA, Brangwynne CP. Quantifying Dynamics in Phase-Separated Condensates Using Fluorescence Recovery after Photobleaching. Biophysical Journal. 2019; 117(7):1285-1300. https://doi.org/10.1016/j.bpj.2019.08.030, doi: 10.1016/j.bpj.2019.08.030.

Tinevez JY, Perry N, Schindelin J, Hoopes GM, Reynolds GD, Laplantine E, Bednarek SY, Shorte SL, Eliceiri KW. TrackMate: An open and extensible platform for single-particle tracking. Methods. 2017; 115:80-90.

Weber CA, Zwicker D, Jülicher F, Lee CF. Physics of active emulsions. Reports on progress in physics Physical Society (Great Britain). 2019; 82(6):064601. http://arxiv.org/abs/1806.09552, doi: 10.1088/1361-6633/ab052b.

Yang P, Mathieu C, Kolaitis RM, Zhang P, Messing J, Yurtsever U, Yang Z, Wu J, Li Y, Pan Q, Yu J, Martin EW, Mittag T, Kim HJ, Taylor JP. G3BP1 Is a Tunable Switch that Triggers Phase Separation to Assemble Stress Granules. Cell. 2020; 181(2):325-345.e28. https://www.sciencedirect.com/science/article/pii/S0092867420303391, doi: https://doi.org/10.1016/j.cell.2020.03.046. 


\section{Methods}

\section{Coacervate assay}

General reagents

Carboxymethyl-dextran sodium salt (CM-Dex, (C6H10O5)n.(COOH), 10-20 kDa, monomer MW = $\left.191.3 \mathrm{~g} \mathrm{~mol}^{-1}\right)$, PolyL-lysine hydrobromide (PLys, (C6H12N2O)n, 4-15 kDa, monomer MW = 208.1 $\mathrm{g} \mathrm{mol}^{-1}$ ) and adenosine 5'-triphosphate disodium salt hydrate (ATP, C10H14N5Na2O13P3, MW $=551.1 \mathrm{~g} \mathrm{~mol}^{-1}$ ) were purchased from Sigma Aldrich. FITCPLys ((C6H12N2O)n.(C21H11NO5S), $\left.25000 \mathrm{~g} \mathrm{~mol}^{-1}\right)$ was purchased from Nanocs, NewYork, USA . Milli Q water was used to prepare aqueous stocks of CM-Dex (1000 mM, pH 8), PLys (200 mM, pH 8) and ATP (100 mM, pH 8). All solutions were stored in the freezer at $-20^{\circ} \mathrm{C}$ until use and the $\mathrm{pH}$ of all stocks was adjusted using a stock solution of $1 \mathrm{M}$ $\mathrm{NaOH}$.

Coacervate preparation

Stock solutions of CM-Dex, PLys and ATP were first diluted to $25 \mathrm{mM}$ and the PLys solution doped with $1 \%$ v/v PLysFITC. Diluted solutions of CM-Dex/PLys or PLys/ATP were then mixed together at a 4:1 volume ratio $(16 \mu 1: 4 \mu 1)$, resulting in the formation of turbid coacervate solutions. Solutions were left to equilibrate for at least 5 min before imaging, up to a maximum of 15 min when larger droplets were desired.

\section{PGL-3 droplets}

PGL-3 was purified and stored as previously described (Saha et al. (2016)). To obtain droplets, $300 \mathrm{mM} \mathrm{KCl} \mathrm{stock}$ protein solution was diluted to the desired concentration, achieving final salt concentrations of 50-180 mM. A small imaging volume was created by using polystyrene beads, resulting in complete droplet sedimentation after less than five minutes. Droplets were imaged immediately to avoid changes in material properties due to ageing (Jawerth et al. (2020)).

\section{Microscopy and FRAP}

\section{Confocal imaging}

Droplets were imaged at midplane by visually defining the focal position with the largest droplet area of the droplet of interest. Images were acquired on an Andor spinning disk confocal microscope equipped with an Andor IX 81 inverted stand, a FRAPPA unit, an Andor iXON 897 EMCCD camera, and a 488nm laser, using a 60x/1.2 U Plan SApo OLYMPUS water objective. Imaging conditions were optimized for minimal bleaching at the required frame rate. Frame rates were optimised for each system: PGL-3, $0.1 \mathrm{~s}<\Delta t<5 \mathrm{~s}$, CMD/PLYS, $\Delta t=0.03 \mathrm{~s}$, PLYS/ATP, $\Delta t=0.07 \mathrm{~s}$.

\section{FRAP}

Droplets were bleached in their entirety by using the minimal FRAP ROI that encompasses the entire droplet. FRAP was performed in three focal planes, equally spaced across the droplet in z-direction, to reduce non-uniform bleaching of the droplet. FRAP rates and dwell times were chosen such that left-over fluorescence intensity above background was smaller than $1 \%$ for PGL-3 and smaller than $15 \%$ for coacervate droplets to maximize bleaching within the droplet while keeping bleaching impact on the droplet environment minimal.

\section{Data analysis}

Azimuthal averaging and normalization

Time-lapse images were cropped with the droplet of interest in the center. An azimuthal average was performed around the center of the droplet to obtain a 1D profile along the radial coordinate with minimal loss of data, using the radialavg function provided by David J. Fischer on Matlab File Exchange. Camera background was subtracted uniformly from the resulting $1 \mathrm{D}$ profiles. The radial intensity profile at the prebleach stage was used for normalization and to correct for optical artefacts that lead to increased fluorescence at the droplet center compared to the droplet-bulk interface. Data close to the droplet interface cannot be used for fitting, since the droplet has an artificially broad boundary due to the point-spread function and likely due to curvature effects. Therefore, on average, the intensity of the ten pixels closest to the boundary were not used for analysis. The droplet boundary was defined as the inflection point of the azimuthally averaged profile in the pre-bleach frame.

Immediately after bleaching a uniform recovery across the entire droplet can be seen, which cannot be spatially resolved even at frame rates $<30 \mathrm{~ms}$. This recovery is fast compared to the recovery by diffusion from the outside for 
all systems under investigation. We thus chose to not account for this uniform recovery in our model and instead start the fitting after a time lag that depends on the system and droplet size. This offset typically consists of less than 5\% of the total pre-bleach intensity. Additionally, bleaching is not complete, resulting in an additional offset above the camera background even immediately after bleaching (see grey lines in Fig. 1).

Photo-bleaching due to continuous imaging was minimal in all droplet types. We thus chose to not account for imaging-induced photo-bleaching, in order to not introduce additional noise due to necessarily occurring fluctuations within the bleach correction.

Extracting experimental boundary conditions

$c_{u}\left(r=R_{-}, t\right)$ was extracted from the intensity profiles as the value at the outermost pixel. In order to speed up fitting and avoid jumps in $c_{u}\left(r=R_{-}, t\right)$, the extracted intensity values were sorted to eliminate small fluctuations.

Fitting of $D_{\text {in }}$ by using experimentally measured boundary conditions (Fig. 1)

The resulting spatio-temporal profiles were fit as described in the main text, using $D_{\text {in }}$ as a single global fit parameter and using $c_{u}\left(r=R_{-}, t\right)$ as described above as the system's time-dependent boundary condition. Fits were performed in MATLAB (Mathworks), using pdepe to solve the PDE and fminsearch for minimizing the squared distance between data and model. Code is available at https://gitlab.pks.mpg.de/mesoscopic-physics-of-life/DropletFRAP.

\section{Numerical solution of Eq. (6)}

Eq. 6 was solved using either pdepde (MATLAB (Mathworks), Figs. 4,5, for spherically symmetric systems) or by using the finite element method via the FENICS environment (Logg et al. (2012)) for arbitrary 3D geometries (Fig. 3). All fits in Figs. 4,5 were performed using fminsearch based on a squared-difference metric. Code is available at https://gitlab.pks.mpg.de/mesoscopic-physics-of-life/frap_theory. 


\section{Appendix 1}

\section{Limit of narrow interfaces}

Here we derive the effective droplet model for our dynamic equation

$$
\begin{aligned}
\partial_{\mathrm{t}} c_{u} & =\nabla \cdot\left[D\left(\phi_{\mathrm{tot}}\right)\left(\nabla c_{u}-c_{u} \frac{\nabla \phi_{\mathrm{tot}}}{\phi_{\mathrm{tot}}}\right)\right] \\
& =-\nabla \cdot \boldsymbol{j}_{\mathrm{u}}
\end{aligned}
$$

by considering the limit of narrow interfaces. In the equation above, $\boldsymbol{j}_{\mathrm{u}}$ denotes the flux of unbleached molecules. Conservation of molecules at the interface implies

$$
\lim _{\ell \rightarrow \epsilon} \boldsymbol{j}_{\mathrm{u}}(R-\ell)=\lim _{\ell \rightarrow \epsilon} \boldsymbol{j}_{\mathrm{u}}(R+\ell),
$$

where $\ell$ is the characteristic size of the interface. Moreover, $\epsilon>0$ is a small but non-zero parameter by which we define the position directly left and right of the interface. In this limit, $\left.\nabla \phi_{\text {tot }}\right|_{R_{ \pm \epsilon}}=0$ directly left and right of the interface. Thus, we obtain Eq. (8c) for narrow interfaces. However, the contribution $D\left(\phi_{\text {tot }}\right) c_{u} \nabla \phi_{\text {tot }} / \phi_{\text {tot }}$ in Eq. (11) that determines the dynamics through the interface implies a Dirichlet boundary conditions for the concentration of unbleached molecules at the interface $r=R$. Since $\nabla \phi_{\mathrm{tot}} / \phi_{\mathrm{tot}} \propto \ell$, we demand

$$
\lim _{\ell \rightarrow 0}\left(\left.\left.\ell c_{u}\right|_{R-\ell} \frac{\nabla \phi_{\mathrm{tot}}}{\phi_{\mathrm{tot}}}\right|_{R-\ell}\right)=\lim _{\ell \rightarrow 0}\left(\left.\left.\ell c_{u}\right|_{R+\ell} \frac{\nabla \phi_{\mathrm{tot}}}{\phi_{\mathrm{tot}}}\right|_{R+\ell}\right)
$$

in the limit of decreasing interface width $\ell$. This condition ensures that the dynamics at the interface remains unchanged for decreasing $\ell$. Parameterizing the interface for example by $\phi_{\mathrm{tot}}(r)=\phi_{\mathrm{out}}^{\mathrm{eq}}[1+(P-1)(1+\tanh (r / \ell)) / 2]$, we find that Eq. (14) leads to Eq. (8d). 


\section{Appendix 2}

\section{Solution of effective droplet model for FRAP}

In this appendix we derive the solution for the two diffusion equations coupled at the interface for a spherically symmetric case; see Eqs. (8). We consider an initial condition with a constant concentration $c(r, 0)=c_{0}$ outside a droplet with radius $R$ and bleaching gives rise to $c(r, 0)=0$ inside the droplet. Performing a Laplace transformation, $\hat{c}(r, s)=\int_{0}^{\infty} d t e^{-s t} c(r, t)$, of Eqs. (8), we find

$$
\begin{array}{ll}
s \hat{c}_{u}(r, s)=D_{\text {in }} \nabla^{2} \hat{c}_{u}(r, s), & \text { for } r<R, \\
s \hat{c}_{u}(r, s)=D_{\text {out }} \nabla^{2} \hat{c}_{u}(r, s)+c_{0}, & \text { for } r \geq R .
\end{array}
$$

where $s$ is the rate parameter of the Laplace transform. The corresponding solutions read

$$
\hat{c}_{u}(r, s)= \begin{cases}\frac{a}{r} \sinh \left(\xi_{\text {in }} r\right), & \text { for } r<R, \\ \frac{b}{r} \exp \left(-\xi_{\text {out }} r\right)+\frac{c_{0}}{s}, & \text { for } r \geq R\end{cases}
$$

where $\xi_{\text {in/out }}=\sqrt{s / D_{\text {in/out }}}$. We have selected the solutions with no radial flux at the origin and a finite concentration at infinity. The remaining unknown constants $a$ and $b$ are determined by the conditions at the interface stated in Eqs. (8). Inside the droplet $r<R$, the solution reads

$$
\hat{c}_{u}(r, s)=\frac{c_{0} P R\left(D_{\text {out }}+R \sqrt{D_{\text {out }} s}\right)}{r s\left(D_{\text {out }}-P D_{\text {in }}+R \sqrt{D_{\text {out }} s}+P R \sqrt{D_{\text {in }} s} \operatorname{coth}\left(R \sqrt{\frac{s}{D_{\text {in }}}}\right)\right)} \frac{\sinh \left(r \sqrt{\frac{s}{D_{\text {in }}}}\right)}{\sinh \left(R \sqrt{\frac{s}{D_{\text {in }}}}\right)} .
$$

Even though it is non-trivial to perform the inverse Laplace transformation of this expression we can obtain some analytical understanding of the behaviour at short and long timescales by considering the asymptotics of large and small $s$. Expanding Eq. (17) for small $s$, we obtain:

$$
\hat{c}_{u}(r, s)=c_{0} P\left[\frac{1}{s}-\frac{\left(2 P \frac{D_{\text {in }}}{D_{\text {out }}}+1\right) R^{2}-r^{2}}{6 D_{\text {in }}}\right]+O\left(s^{1 / 2}\right) .
$$

This relationship shows that for small $s$, the rescaled concentration $\hat{c}_{u}(r, s) / P$ is affected by $D_{\text {out }}$ only via the $P D_{\text {in }} / D_{\text {out }}$ combination. Conversely, for large $s$, the leading contribution features the term

$$
\hat{c}_{u}(r, s) \sim \frac{c_{0} P R}{r s}\left(1+P \sqrt{\frac{D_{\text {in }}}{D_{\text {out }}}}\right)^{-1} e^{(r-R)} \sqrt{\frac{s}{D_{\text {in }}}}
$$

showing that for short times the system is influenced by $D_{\text {out }}$ via the ratio $P \sqrt{D_{\text {in }} / D_{\text {out }}}$. Note that for these short times the solution is well approximated by that of a one-dimensional system. These long and short time behaviors are confirmed by the numerical solutions plotted in Appendix 2 Fig. 1, which show that the evolution of the rescaled concentrations match at short times, if $P \sqrt{D_{\text {in }} / D_{\text {out }}}$ is kept constant but start to deviate for longer times. Conversely, at longer times the dynamics are invariant if $P D_{\text {in }} / D_{\text {out }}$ is kept constant except for a shift due to a short timescale transient. 
bioRxiv preprint doi: https://doi.org/10.1101/2021.03.08.434288; this version posted March 8, 2021. The copyright holder for this preprint (which was not certified by peer review) is the author/funder, who has granted bioRxiv a license to display the preprint in perpetuity. It is made available under aCC-BY-NC 4.0 International license.
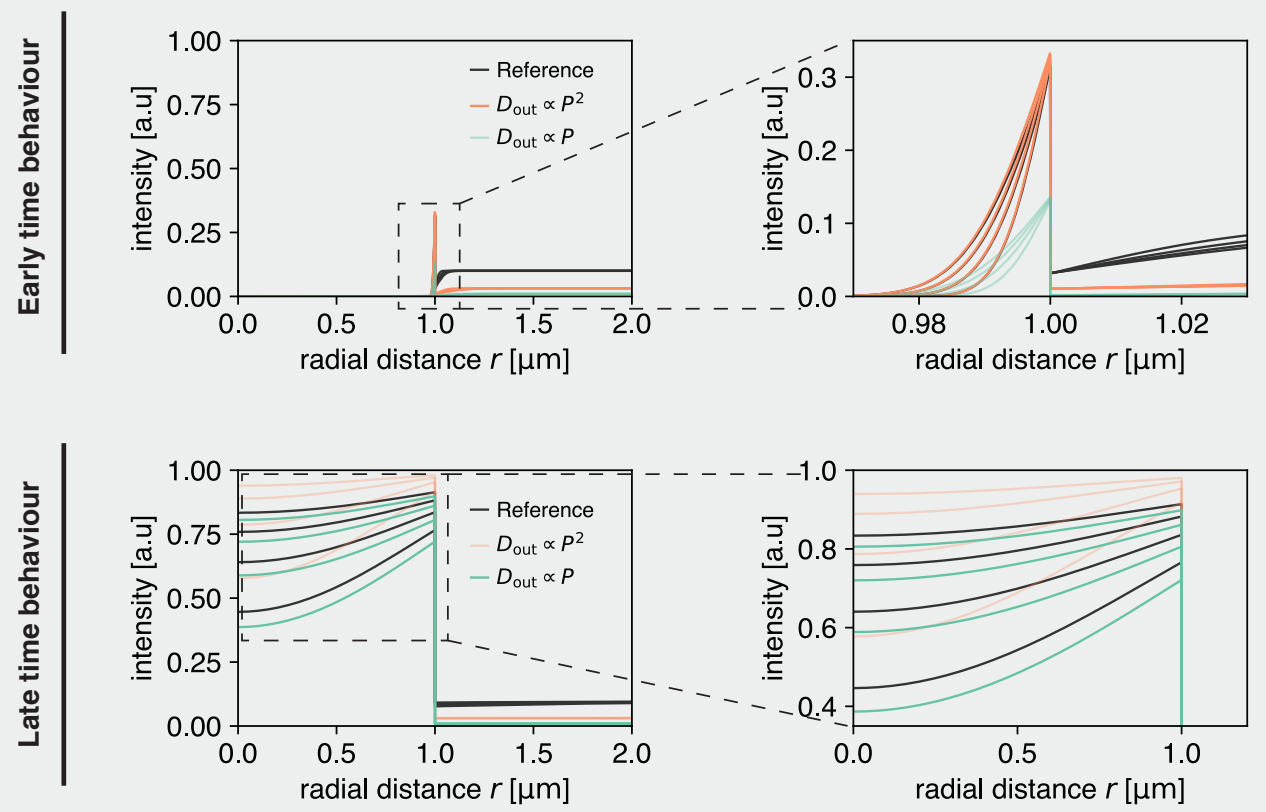

Appendix 2 Figure 1. Solution to dynamics inside droplets is unique, but early and late time behaviour can be approximated by different scaling regimes. (Top) Early time behaviour of numerical solutions. If a parameter set is chosen ('Reference'), at early times this can be approximately recovered by scaling $D_{\text {out }} \propto P^{2}$ (orange). A linear scaling does not recover the same inside dynamics at early times (green). (Bottom) Late time behaviour of numerical solutions. At late times the reference dynamics can be approximately recovered by scaling $D_{\text {out }} \propto P$ (green). Meanwhile, $D_{\text {out }} \propto P^{2}$ does not recover the reference inside dynamics (orange). 